\title{
Transformación digital en Ecuador: la pandemia como acelerador del ecosistema
}

\author{
Daniel F. López Jiménez \\ daniell@uhemisferios.edu.ec \\ https://orcid.org/0000-0002-9163-8004 \\ Doctor en Economía de la Empresa \\ por la Universidad Rey Juan Carlos (ES) \\ Vicerrector Académico de la Universidad Hemisferios (EC) \\ Quito - Ecuador \\ Juan Pablo del Alcázar Ponce \\ Jpdelal@gmail.com \\ https://orcid.org/0000-0002-5444-1691 \\ Doctorando en Gestión de Educación Superior \\ por la Universidad de Palermo (AR) \\ Profesor Maestría Comunicación Estratégica - \\ Universidad Hemisferios \\ Quito - Ecuador
}

\section{RESUMEN}

El presente informe de investigación presenta las evidencias de la transformación digital que ha tenido el Ecuador en los últimos años, desde la perspectiva del crecimiento del consumo de Internet y las redes sociales. El estudio analiza las métricas de diferentes fuentes especializadas, en articulación con los estudios del World Internet Project desde el enfoque teórico de la ecología humana de la comunicación del Ecuador, significando sus particularidades endógenas y exógenas de su sociedad, en las que se sustenta el cambio acelerado que ha tenido el país, alejado de la normal evolución tecnológica y presentándose como un proceso acelerado de transformación digital.

Palabras Claves: transformación digital; Ecuador; consumo; medios; digitales; ecología; comunicación 


\title{
Digital transformation in Ecuador: the pandemic as an accelerator of the ecosystem
}

\begin{abstract}
This research report presents the evidence of the digital transformation that Ecuador has undergone in recent years, from the quantitative perspective of digital media consumption and the qualitative particularity of business strategies focused on taking advantage of the new dynamics of consumer habits of netizens. The study analyzes the metrics of different specialized sources, in conjunction with the studies of the World Internet Project from the theoretical approach of the human ecology of Ecuador's communication, meaning its endogenous and exogenous particularities of its society, in which the change is based. accelerated that the country has had, away from the normal technological evolution and presenting itself as an accelerated process of digital transformation.
\end{abstract}

Keys Words: digital transformation; Ecuador; consumption; media; digital; ecology; communication

. Artículo recibido: 02 Setiembre. 2021 Aceptado para publicación: 30 Setiembre. 2021 Correspondencia: santiagou@ uhemisferios.edu.ec Conflictos de Interés: Ninguna que declarar 


\section{INTRODUCCIÓN}

Los últimos 10 años fueron trascendentales para el Ecuador en su "transformación" tecnológica, concebida esta como la máxima aceleración posible del cambio, en el que la propia esencia constitutiva puede verse amenazada, en este caso, la particularidad propia de su sociedad. Este fenómeno ha sido observado inicialmente por el estudio longitudinal cualitativo del World Internet Project -WIP-Ecuador, y en los últimos años, especialmente por diferentes plataformas y centros de investigación orientadas a estudios de métricas cuantitativas Facebook, Google, Twitter, We are social, App Annie y Alexa, Statista, Wordstream, Grouppm Nextyear Media Forecasts, entre otros, y centros de análisis de datos especializados como Mentinno, Statcounter, Semrush.com, Similar Web, Kantar Ibope Media Ecuador y la Cámara Ecuatoriana de Comercio Electrónico, Adcuality, Superintendencia de Compañías Ecuador, entre otras.

En el presente artículo, esta transformación se analiza desde los datos del año -2020- el primer año de la pandemia del COVID-19-, a partir de hábitos de consumo de nuevos medios digitales en relación con la usabilidad, el consumo de los cibernautas y su interrelación en redes sociales, páginas webs, y aplicaciones, suministrados en tiempo real por las métricas cuantitativas de las plataformas citadas en el párrafo anterior.

El enfoque que se utiliza para la argumentación del fenómeno de transformación tecnológica en Ecuador, parte del modelo de Ecología Humana de la Comunicación, publicado en 2016 en la revista Comunicación y Sociedad de la Universidad de Navarra, en el que se identifican los aspectos endógenos y exógenos de los componentes mediáticos de la sociedad, en compañía de los colegas Javier Odriozola y Juan David Bernal.

Los datos analizados evidencian el crecimiento de la confianza de los cibernautas consumidores en las plataformas comerciales, consolidando un nuevo mercado digital, que aunque fue detonado por la pandemía durante el año 2020, ya había iniciado su transformación temprana desde los últimos 10 años, y en el que el ecosistema tecnológico encontró un ambiente ideal de desarrollo a partir de los hábitos de consumo de los nuevos medios de comunicación digital, la cual permitieron una aceleración sin precedentes, hacia el establecimiento del E-commerce, definiendose como una nueva realidad posible de la nueva normalidad, al lado de la Teleeducación y el Teletrabajo. 


\section{Estado de la cuestión}

A partir de marzo 12 de 2020, el Comité de Emergencias de Ecuador COE ordenó el confinamiento total de la sociedad ecuatoriana, con excepción de las instituciones prestadoras de salud, y algunos sectores estratégicos de producción. Dicha ordenanza, autorizaba el teletrabajo y la teleeducación.

Los estudios cualitativos del consumo de Internet y su tipificación en los diferentes países predominaron en la última década, principalmente por el World Internet Project- WIP, el cual nació en el Centro para la Comunicación Política de la Universidad de California-UCLA-, en colaboración con la Escuela de Ciencias de la Comunicación UNT de Singapur, y el Observatorio Italiano de Internet, de la Universidad Bocconi de Milán. En la actualidad, el proyecto se dirige desde el Centro para el Futuro Digital de la Escuela de Comunicación “Annenberg” de la Universidad del Sur de California-USC-, por el fundador y actual director Jeffrey Cole. Para el año 2020 el proyecto contaba con la participación de 34 países, inclusive Ecuador, con el liderazgo del Centro de Altos Estudios de Internet, de la Facultad de Comunicación y Tecnologías de la Información de la Universidad de Los Hemisferios.

Simultáneamente, el proyecto del World Stats, en la última década adelanta estudios cuantitativos de penetración de Internet en 246 países con actualización mensual de datos estadísticos. La línea de investigación sobre los hábitos de consumo en el Ecuador, además del proyecto WIP- Ecuador, se destaca el estudio “La Generación Interactiva en Ecuador” (Bringué \& Sádaba, 2011), Por otra parte, el Ministerio de Telecomunicaciones -MINTEL- publica anualmente el "Informe Anual de Estadísticas de Tecnologías de la Información y las Comunicaciones”, y más recientemente el Instituto Nacional de Estadísticas y Censos, realiza estudios de tecnologías de información y comunicación, aun con metodologías cualitativas.

Por su parte, y recientemente, el portal Worldmeter ofrece datos estadísticos de consumo de Internet, en tiempo real, lo que ha generado, cierta desconfianza entre la comunidad académica del campo de la comunicación digital. Sin embargo, aporta datos estadísticos de tendencia sobre la economía de la producción tecnológica, susectibles de utilizar en estudios cualitativos de prospectiva.

En la actualidad, los estudios cualitativos han venido fortaleciendose con estudios cuantitativos, producidos por los departamentos de estadística de cada país, y en especial por los sistemas de medición métrica de cada una de las plataformas tecnológicas de comunicación digital, de acuerdo con la naturaleza del servicio, y de conformidad con la base de datos que se produce a partir del registro de los usuarios. En este sentido, las métricas se desarrollaron en torno a la 
utilización de los nuevos medios de comunicación digital, redes sociales, marketing empresarial y político, utilización de software, visitas de sitios webs, videojuegos, apuestas, correo electrónico, etc., de tal manera que motores de búsqueda como Google, y redes sociales como Facebook y Twitter, entre otros, asi como sistemas de medición como App Annie y Alexa, dedicaron un segmento del modelo de su negocio a la medición de los hábitos de consumo de las cibernautas, ahora entendidos como Ciber consumidores y prosumidores, que les permitiera identificar comportamientos y predecir decisiones de cada persona, a partir de la generación de perfiles particulares, lejanos de las predecesoras estrategias del marketing, basadas en la segmentación, ahora basadas en la fragmentación de audiencias, o mejor, a la personalización del consumo, tal como lo realizan centros de investigación dedicados a la inteligencia de mercados digitales, como Menntino en Ecuador, con la dirección de su investigador principal Juan Pablo Benalcázar, quien a través de cruces de datos de diferentes métricas ha venido proporcionando fuentes de información para la toma de decisiones empresariales.

Producto de las investigaciones cualitativas se intentó teorizar sobre nuevas categorías universales que explicaran la comunicación social. En esta línea han sido significativos los aportes a la ciencia de la Comunicación, de la Escuela Funcionalista norteamericana, Laswell (1948), Lazarsfel y Stanton (1949), Shannon y Weaver (1949), Hovland et al. (1949), Defleur (1964), Lippman (1922), Sears et al. (1973), Petty y Priester (1981), de la Escuela Hermeneútica o del Interaccionismo Simbólico italiana, Eco (1986), Barthes (1972), de los Estudios Culturalistas latinoamericanos, Barbero, Rey (1999), de la Escuela de la Insdustrias Culturales de Frankfurt, Habermas (1989), Adorno y Horkeimer (1972), Mattelart (1997), Marcuse (2001), la Escuela de los Mass Media, Newman (1982), McQuail (1983), McCombs (1993) y Levy (1977). Sin embargo, ninguna de estas escuelas se acercó a una explicación suficiente de los fenómenos comunicativos que se desarrollaron desde la llegada de Internet en la década de los años 90 s.

La Escuela que, de alguna manera, previó gran parte de los efectos globalizantes de los medios de comunicación digital fue la Escuela Canadiense promovida por MacLuhan. Contrariamente, su enfoque determinista, en el que, -la tecnología determina la cultura-, cerró la puerta al estudio de las particularidades de cada comunidad, de cadas sociedad, de cada país. Y, su enfoque globalizante, desvirtuó cualquier relevancia significativa de la localidad, a pesar de la refundación de dicha escuela, y su viraje hacia una visión ecosistémica denominada la Media Ecology, Altheide, (1994) y Krekchove, (2002), Cali (2012) quienes por primera vez utilizaron el término Ecología de la Comunicación. Sin embargo, la incapacidad de explicar los nuevos fenómenos de comunicación digital, Scolari (2008) propició la búsqueda de nuevas categorías 
teóricas que dieran explicación, más allá de las tendencias globales, a las particularidades locales.

De tal manera que se propuso el enfoque de la "Ecología Humana de la Comunicación” López, (2016), en la que, desde una visión interdisciplinaria de la realidad social comunicativa, se pudiera comprender una realidad desde diferentes miradas disciplinarias, como la antropología, la política, economía, sociología, psicología social, historia, y todas aquellas disciplinas necesarias, propendiendo porque es la cultura la que determina la tecnología, y no lo contrario. La Ecología Humana de la Comunicación, busca la explicación de los fenómenos comunicativos locales desde un enfoque heurístico del ecosistema social mediático, en los que se destacan los estudios pioneros de Carpenter, (1956), Mumford, (1967), Langer (1967), Ellul (1967), McLuhan (1970) (ed.1996), Havelock (1981), Eisenstein (1983), Cabrera, (1988), Innis (1999), Postman (1988), (2000), y Schofield (2009). Es necesario señalar que este enfoque teórico, surgió gracias a la investigación longitudinal del World Internet, Project- capítulo Ecuador. En el que cada año, se reunían los diferentes grupos de investigación asociados al proyecto de cada uno de los países miembros a compartir los datos referentes a la tipificación del consumo de Internet nacional. En este ejercicio registrado en la publicación de diversos artículos de López, Odriozola, Bernal, (2016), López, Callejo, Cajiao (2013), López, Eguiguren (2011) y López, (2010), se advertía sobre el acelerado y exponencial crecimiento del consumo de Internet en el Ecuador, el cual, pasó de un consumo del 25\% de su población en el año 2009, a un $80 \%$ en el año 2016, fenómeno inexplicable desde las mismas variables del estudio en otros países de la zona como Colombia, Chile, Argentina y México, Dyjament (2010).

\section{Ecuador como ecosistema de Internet}

Ecuador es un país singular por sus características como se ha señalado por los estudios de la Ecología Humana de Comunicación (2016). Cuenta con una población estimada de 17.510.643 (proyecciones INEC, 2020). Cerca del 50\% de su territorio se ubica en la región sierra-costa, y su otro 50\% en la región amazónica. En la sierra-costa, se ubica el 95\% de la población. Del total de su población, el 80,1\%, (14,25 millones) tienen acceso a Internet para el año 2020, según los datos del estudio de Mentinno (2020), el cual, al igual que el WIP, consideran la variable de multiusuario por conexión, a diferencial INEC, que solo determina la cantidad de usuarios por conexión de Internet, en relación de 1:1, mientras que los multiusuarios se estiman entre 1:2, o 1:1,5, que para el efecto ecuatoriano es de 1:1,5. De esta cantidad de usuarios, 14 millones utilizan redes sociales, (78,7\%). Existen 14,88 millones de líneas moviles de telefonía 
celular $(83,6 \%)$. Del total de usuarios de redes sociales, la mayor participación se encuentra en Guayaquil con un 15\%, seguido por Quito por un 12\%, Cuenca 4\%, Ambato 3\%, Santo Domingo 3\%, Riobamba, Machala, Manta, Portoviejo y Loja, cada uno con 2\%. El restante $53 \%$ se distribuye en las demás ciudades de menos de 120 habitantes (Facebook Audience Insights, 2021).

La dinámica que muestra el país en materia de acceso a Internet tiene especiales causas que a lo largo de los últimos años han sido motivadas principalmente por las políticas de gobierno de modernización de los centros escolares y la dotación de tecnología con acceso a Internet. Así mismo las campañas de Internet móvil para las poblaciones más alejadas de la geofrafía nacional, como también la disposición de Internet gratuito en parques en diferentes ciudades, centros comerciales, edificios públicos, de transporte etc. (López, Odriozola, Bernal, 2016).

\section{Metodología y hallazgos}

El presente estudio analiza las métricas de fuentes especializadas, referentes en la medición longitudinal de datos cuantitativos en el Ecuador, en relación con los hábitos de consumo de Internet en redes sociales, y su relación asociativa comercial, más conocida como e-commerce en el primer año de la Pandemia 2020. Las métricas analizadas fueron Facebook, Google, Twitter, We are social, App Annie y Alexa, Statista, Wordstream, Grouppm Nextyear Media Forecasts, y los centros de análisis de datos especializados Statcounter, Semrush.com, Similar Web, Kantar Ibope Media Ecuador, la Cámara Ecuatoriana de Comercio Electrónico, Adcuality y Superintendencia de Compañías Ecuador. Las métricas fueron seleccionadas por la confiabilidad de sus metodologías cuantitativas, soportadas en bases de datos propias y por la utilidad explicita del tratamiento de su big data, para investigadores y analistas especializados. El análisis se realizó sobre los datos obtenidos en el mes de enero de 2021, luego del cierre estadístico del año 2020. Las variables del estudio fueron: usuarios de Internet, comportamiento y niveles de digitalización, ranking y perfiles de usuarios de redes sociales, principales sitios web y aplicaciones móviles, E- Commerce, inversión en medios digitales, medios de comunicación locales, streaming y universidades, gamers y juegos en línea. Sin embargo, y para el efecto, en el presente informe se analiza el estado de los usuarios de Internet, en asociación con su comportamiento y niveles de digitalización.

\section{Hallazgos}

En relación con los Usuarios de Internet en el Ecuador, como se mencionó en el numeral 2.1. del presente informe de investigación, el 80,1\%, (14,25 millones) tienen acceso a Internet en 2020. De esta cantidad de usuarios, 14 millones utilizan redes sociales. Existen 14,88 millones de líneas móviles de telefonía celular. Adicionalmente, el 59\% de los usuarios de Internet son 
mayores de 24 años, y el 98\% de los usuarios menores de 24 años, tienen interrelación en redes sociales vía dispositivos móviles.

Frente al comportamiento y los niveles de digitalización de los internautas en el Ecuador, el estudio pudo determinar que, en el año 2020, producto del confinamiento dispuesto por las autoridades nacionales, los hábitos de consumo de las personas se enfocaron principalmente en una nueva agenda mediática digital como se observa en la Tabla I.

Tabla I. Categorías y actividades de cibernautas en Ecuador 2020

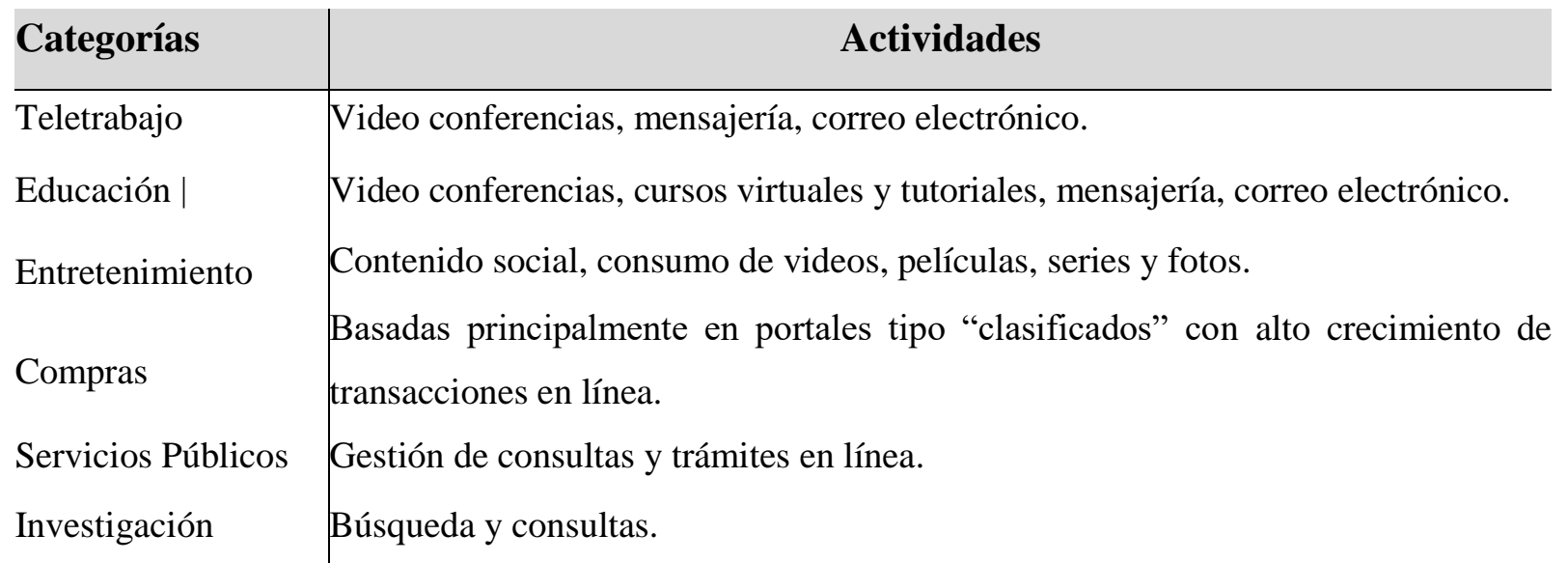

\section{Fuente: Elaboración propia - Mentinno}

El teletrabajo, la teleeducación, el entretenimiento, las compras, el pago de servicios públicos y la investigación escolar, en su orden se convirtieron en los principales motivos de utilización de Internet durante el primer año de la pandemia 2020, cambiando el orden mantenido en los últimos 5 años, donde aparecía el entretenimiento mediante la utilización de redes sociales y aplicaciones musicales y de televisión. 
Tabla II. Tiempo diario en sitios web de mayor visitas enero-dic 2020

\begin{tabular}{l|c|c} 
Sitio & \multicolumn{2}{|c}{ Promedio por día } \\
\hline Google & $15: 44 \mathrm{~min}$ & 17,1 páginas \\
YouTube & $17: 10 \mathrm{~min}$ & 9,78 videos \\
Facebook & $18: 50 \mathrm{~min}$ & 8,83 páginas \\
Mercado libre & $6: 10 \mathrm{~min}$ & 6,67 páginas \\
\hline
\end{tabular}

Fuente: Elaboración propia con base en métrica de Alexa top sites Ecuador. (01-2021)

En relación con el tiempo diario que las personas en Ecuador dedicaron en promedio, a la visita de sitios web en el último año, en la Tabla II se puede determinar la utilización del tiempo en relación con las visitas a páginas interiores y videos. Esta frecuencia es significativa, en cuanto evidencia el grado de interactividad que podría estar generándose en estas plataformas.

Tabla III. Principales búsquedas en Google enero- dic 2020

\begin{tabular}{c|c|c} 
Número & Búsqueda & Índice \\
\hline 1 & Traductor & 100 \\
2 & Facebook & 81 \\
3 & WhatsApp & 70 \\
4 & YouTube & 52 \\
5 & Web WhatsApp & 51 \\
6 & Google & 41 \\
7 & Hotmail & 39 \\
8 & Pichincha & 28 \\
9 & Gmail & 26 \\
10 & Juegos & 25 \\
\hline
\end{tabular}

Fuente: Elaboración propia con base en métrica de Google Trends Ecuador. Últimos 12 meses. Enero 2 de 2021

En cuanto a la tipificación de las búsquedas en Google, en la Tabla III, se puede observar cómo, este motor de búsqueda, en virtud de su función principal, sirve como un puente comunicativo entre las necesidades de las personas y la tecnología virtual de Internet, configurándose más como un canal que como un medio de comunicación. El portal de Traductor de Google se ha convertido en una posibilidad de acceso a la información en otras lenguas, especialmente el inglés, de aquellas personas que no dominan este idioma. 
Tabla IV. Principales búsquedas cualitativas en Google sobre "cómo, que, donde" enerodic 2020

¿Cómo?

¿Cómo saber si estoy embarazada?

¿Cómo hacer un ensayo?

¿Cómo recuperar mensajes eliminados de WhatsApp?

¿Cómo eliminar una cuenta de Facebook?

¿Cómo descargar among us para pc?

¿Cómo saber si soy beneficiaria del bono? ¿Cómo bajar de peso?

¿Cómo dibujar un perro?

¿Cómo tomar captura de pantalla en pc? ¿Cómo poner fondo en zoom?
¿Qué?

¿Qué paso con Instagram?
¿Qué es endémico?
¿Qué placas circulan hoy?
¿Qué es alerta naranja en Ecuador?
¿Qué es cultura?
¿Qué se celebra el 28 de diciembre?
¿Qué significa uwu?
¿Qué significa endémico?
¿Qué es democracia?
¿Qué es alerta naranja?

¿Dónde?

¿Dónde registramos los asientos contables?

¿Dónde me toca votar? ¿Dónde me toca sufragar?

¿Dónde nació Jesús?

¿Dónde votar 2021?

¿Dónde se originó el COVID? ¿Dónde pagar multas ANT? ¿Dónde podemos encontrar comida rápida?

Fuente: Elaboración propia con base en métrica de Mundomundo

Frente a las principales búsquedas en Google que realizan las personas en Ecuador, se advierte que están orientadas por tres categorías iniciales de pregunta: cómo, qué y dónde. En las Tablas IV y V, se advierte una relación directa, con la nueva agenda de usabilidad de Internet, como se había advertido en la Tabla I. Llama la atención, que los motivos de consulta guardan una relación muy estrecha con la realidad social del país, en la también puede advertirse que Google se ha convertido más que en un motor de búsqueda, en un repositorio útil de información para el desarrollo de la vida de las personas.

Tabla V. Principales búsquedas cualitativas en Google sobre "cómo, que, donde" enerodic 2020

\begin{tabular}{l|l}
\hline ¿Cómo convertirse? & ¿Cómo ser? \\
\hline ¿Cómo convertirse en sirena? & $\begin{array}{l}\text { ¿Cómo ser una persona ética en los diferentes } \\
\text { aspectos de la vida? }\end{array}$ \\
\hline ¿Cómo convertirse en vampiro? & ¿Cómo ser un Latin lover? \\
\hline ¿Cómo convertirse en sirena en luna llena? & ¿Cómo ser feliz? \\
\hline ¿Cómo convertirse en un hombre lobo? & ¿Cómo ser un buen líder? \\
\hline ¿Cómo convertirse en hacker en free fire? & ¿Cómo ser un youtuber? \\
\hline ¿Cómo convertirse en súper sayayín? & ¿Cómo ser un hacker en free fire? \\
\hline ¿Cómo convertirse en una persona optimista? & $\begin{array}{l}\text { ¿Cómo ser creativo para generar ideas para } \\
\text { cambiar nuestro mundo? }\end{array}$ \\
\hline ¿Cómo convertirse en youtuber? & ¿Cómo ser socio de Garena? \\
\hline ¿Cómo convertirse en animales en minecraft? & ¿Cómo ser un buen ciudadano? \\
\hline
\end{tabular}

Fuente: Elaboración propia con base en métrica de Mundomundo 
Por su parte, YouTube, se ha convertido en el repositorio de entretenimiento audiovisual, ya no solo de videos musicales, sino de películas, documentales, series, noticieros y resúmenes informativos, convirtiéndose no solo en fuente de entretenimiento sino a hora en fuente de información, tal como lo refleja la Tabla VI.

Tabla VI. Principales búsquedas en YouTube enero-dic 2020

\begin{tabular}{c|l|c} 
Número & \multicolumn{1}{|c}{ Búsqueda } & Índice \\
\hline 1 & Películas & 100 \\
2 & Películas & 91 \\
3 & Música & 84 \\
4 & Películas completas en español & 69 \\
5 & La rosa de Guadalupe & 42 \\
6 & TikTok & 38 \\
7 & La rosa de Guadalupe & 36 \\
8 & Canciones & 35 \\
9 & Salsa & 32 \\
10 & Películas completas en español latino & 26 \\
\hline
\end{tabular}

Fuente: Elaboración propia con base en métrica de Google Trends Ecuador. Últimos 12 meses.

Frente al uso y perfiles de usuarios de Internet, se observa en la Tabla VII, el número en millones de usuario que tienen cuentas en las 9 principales redes sociales. Este número, en cada una de ellas, es significativo para la interacción comunicativa, dinámica que han venido aprovechando los anunciantes de publicidad, colonizando nuevos escenarios en Internet.

Tabla VII. Audiencias redes sociales

\begin{tabular}{l|c|llll} 
Redes & $\begin{array}{c}\text { Volumen en } \\
\text { millones de } \\
\text { usuarios }\end{array}$ & & & & \\
\hline Facebook & $13,300.000$ & $2,500.000$ & Administradores & $1,500.000$ & Marketplace \\
Google/YouTube & $10,500.000$ & & & & \\
Instagram & $5,200.000$ & $3,800.000$ & Stories & & \\
LinkedIn & $2,900.000$ & & & & \\
Spotify & $2,900.000$ & & & & \\
TikTok & $2,300.000$ & & & & \\
Twitter & $1,000.000$ & & & & \\
Pinterest & $1,200.000$ & & & & \\
Snapchat & 259.000 & & & & \\
\hline
\end{tabular}

Fuente: Elaboración propia con base en métrica de Formación Gerencial. Facebook Ads, Google Ads, Twitter Ads, LinkedIn Ads, Snapchat Ads. Información Spotify y Pinterest

Respecto a la mensajería instantánea se advierte en la Tabla VIII, como la plataforma Telegram, posiblemente, y como respuesta a los diferentes rumores sobre la inseguridad de la información personal que viene manejando WhatsApp. En conjunto, es llamativo que las personas utilizan, 
no solo una, sino varias aplicaciones de mensajería, y que potencialmente también reflejan un nuevo ambiente comunicacional a distancia, que significativamente arroja indicios del mejoramiento de la calidad de vida de las personas, en cuanto a la posible disminución de la incertidumbre que supone la convivencia en la distancia física.

\section{Tabla VIII. Mensajería instantánea}

\begin{tabular}{l|c|cc}
\multicolumn{1}{c|}{ Redes } & $\begin{array}{c}\text { Volúmen en millones de } \\
\text { usuarios }\end{array}$ & \\
\hline WhatsApp & $13,300.000$ & $2,500.000$ & Administradores \\
Messenger & $10,500.000$ & $3,800.000$ & Stories \\
Telegram & $5,200.000$ & & \\
Signal & $2,900.000$ &
\end{tabular}

Fuente: Elaboración propia con base en métrica de Mentinno - Formación Gerencial

El crecimiento de Telegram se evidencia en que durante el año 2020 fue la aplicación más descargada en Ecuador, como se evidencia en la Tabla IX. Llama la atención que de las 13 aplicaciones de mayor descarga en el país, las 13 son redes sociales, evidenciando un fenómeno de necesidad comunicativa entre los usuarios durante el primer año de la pandemia, en el que el confinamiento se materializó en la no presencia y el distanciamiento físico, no así en la comunicación social, gracias a las nuevas tecnologías de información y comunicación TIC, conformadas por Software, Hardware y Redes, y que para el efecto, se refleja en los la disponibilidad de Internet, de los dispositivos móviles, computadores, y tabletas, y la usabilidad de las redes sociales.

Tabla IX. Principales descargas de App, redes sociales, mensajería

\begin{tabular}{c|l} 
Lugar & \multicolumn{2}{|c}{ APP-Redes sociales } \\
\hline 1 & Telegram \\
2 & Signal \\
3 & WhatsApp \\
4 & TikTok \\
5 & Facebook \\
6 & Instagram \\
7 & Messenger \\
8 & YouTube \\
9 & Snapchat \\
10 & Pinterest \\
11 & Twitter \\
12 & Twitch \\
13 & LinkedIn \\
\hline
\end{tabular}

Fuente: Elaboración propia con base en métrica de App Annie Top Apps Ecuador 
Facebook sigue siendo la mayor red social que se utiliza en el país, con 13,300 millones de usuarios, principalmente por el grupo de personas entre 18 y 44 años; seguida por LinkedIn con 2,943 millones de usuarios, principalmente entre 25 a 34 años, en correspondencia con la edad productiva de profesionales universitarios; la recién creada TikTok, con 2,300 millones, principalmente por personas entre 18 y 34 años, Twitter con 1,000 millón, mayormente entre 18 a 44 años, y Snapchat con 260 mil usuarios, principalmente entre 18 y 24 años de edad. (ver Tabla X.) Cada una de estas redes sociales ofrece una lógica comunicativa, a partir de la interacción social de intereses particulares, conformadas por tribus o comunidades digitales, comprendidas por el interés compartido, de información, entretenimiento, comercio, opinión, entre otras.

Tabla X. Usuarios de redes sociales por edades

\begin{tabular}{c|cc|cc|cc|cc|cc|c|c|}
\multicolumn{2}{|c|}{ Facebook } & \multicolumn{2}{c|}{ Instagram } & \multicolumn{2}{c|}{ LinkedIn } & \multicolumn{2}{c|}{ Twitter } & \multicolumn{2}{c|}{ TikTok } & \multicolumn{2}{c|}{ Snapchat } \\
Edad & Usuarios & $\%$ & Usuarios & $\%$ & Usuarios & $\%$ & Usuarios & $\%$ & Usuarios & \% & Usuarios & $\%$ \\
\hline 13 a 17 & 1.100 & $8 \%$ & 390 & $8 \%$ & & & 77 & $8 \%$ & 230 & $16 \%$ & 87 & $34 \%$ \\
18 a 24 & 3.600 & $27 \%$ & 1.700 & $32 \%$ & 830 & $28 \%$ & 363 & $36 \%$ & 790 & $55 \%$ & 113 & $43 \%$ \\
25 a 34 & 3.900 & $29 \%$ & 1.600 & $31 \%$ & 1800 & $61 \%$ & 328 & $33 \%$ & 690 & $48 \%$ & 42 & $16 \%$ \\
35 a 44 & 2.300 & $17 \%$ & 800 & $15 \%$ & 290 & $10 \%$ & 133 & $13 \%$ & 330 & $23 \%$ & 15 & $6 \%$ \\
45 a 54 & 1.300 & $10 \%$ & 350 & $7 \%$ & & & 61 & $6 \%$ & 150 & $10 \%$ & 3 & $1 \%$ \\
55+ & 1.100 & $8 \%$ & 240 & $5 \%$ & 23 & $1 \%$ & 38 & $4 \%$ & 95 & $7 \%$ & & \\
No iden & & & 120 & $2 \%$ & & & & & 15 & $1 \%$ & & \\
Total & $\mathbf{1 3 . 3 0 0}$ & $\mathbf{1 0 0}$ & $\mathbf{5 . 2 0 0}$ & $\mathbf{1 0 0 \%}$ & $\mathbf{2 . 9 4 3}$ & $\mathbf{1 0 0 \%}$ & $\mathbf{1 . 0 0 0}$ & $\mathbf{1 0 0 \%}$ & $\mathbf{2 . 3 0 0}$ & $\mathbf{1 6 0 \%}$ & $\mathbf{2 6 0}$ & $\mathbf{1 0 0 \%}$ \\
\hline
\end{tabular}

Fuente: Elaboración propia con base en métrica de App Annie Top Apps Ecuador, Facebook Ads, LinkedIn Ads, Twitter Ads, Snapchat Ads

En relación con el tipo de sexo de los usuarios de las redes sociales en Ecuador, es significativo las diferencias de uso que presentan cada una de ellas, si consideramos que la población del país guarda una proporción de $\mathrm{M=51 \%}$ y $\mathrm{H}=49 \%$ con la estructura mundial de sus habitantes. En este sentido, Instagram, TikTok y Snapchat tienen una mayor prevalencia de uso por parte de las mujeres, mientras que Facebook, LinkedIn y especialmente Twitter son de mayor uso por los hombres. No se podría dar una explicación a priori o con algún grado de inferencia sobre este particular, lo que supondría estudios cualitativos para comprender este comportamiento. (Ver tabla XI.) 
Tabla XI. Usuarios en redes sociales por género

\begin{tabular}{l|cc} 
Red Social & Hombre & Mujer \\
\hline Facebook & $52 \%$ & $48 \%$ \\
Instagram & $46 \%$ & $54 \%$ \\
LinkedIn & $55 \%$ & $45 \%$ \\
Twitter & $60 \%$ & $40 \%$ \\
TikTok & $46 \%$ & $54 \%$ \\
Snapchat & $38 \%$ & $62 \%$
\end{tabular}

Fuente: Elaboración propia con base en métrica de Facebook Ads, LinkedIn Ads, Snapchat Ads

Llama la atención la preocupación que demuestran los estudiantes universitarios en la utilización de la red social Linkedin, en la que se advierte que las redes sociales, pueden ofrecer alternativas informativas útiles para una determinada comunidad virtual, como se observa en la Tabla XII.

Tabla XII. Ranking Estudiantes Universitarios en LinkedIn Ecuador 2020

\begin{tabular}{l|cc}
\multicolumn{1}{c|}{ Universidad } & Usuarios & $\%$ \\
\hline Universidad de Guayaquil & 96000 & $16,3 \%$ \\
Universidad Central & 77000 & $13,1 \%$ \\
Pontificia Universidad Católica del Ecuador & 34000 & $5,8 \%$ \\
Universidad Católica Santiago de Guayaquil & 32000 & $5,4 \%$ \\
Universidad Técnica Particular de Loja & 32000 & $5,4 \%$ \\
Escuela Superior Politécnica del Litoral & 33000 & $5,6 \%$ \\
Universidad Politécnica Salesiana & 33000 & $5,6 \%$ \\
Escuela Superior Politécnica del Ejército & 31000 & $5,3 \%$ \\
Escuela Politécnica Nacional & 22000 & $3,7 \%$ \\
Universidad Tecnológica Equinoccial & 23000 & $3,9 \%$ \\
Universidad de Las Américas & 20000 & $3,4 \%$ \\
Universidad San Francisco de Quito & 19000 & $3,2 \%$ \\
Universidad Técnica de Ambato & & $0,0 \%$ \\
Universidad de Cuenca & 17000 & $2,9 \%$ \\
Escuela Superior Politécnica de Chimborazo & 16000 & $2,7 \%$ \\
Universidad Laica Vicente Rocafuerte & 16000 & $2,7 \%$ \\
Universidad Nacional de Loja & 14000 & $2,4 \%$ \\
Universidad del Azuay & 9500 & $1,6 \%$ \\
Universidad Católica de Cuenca & 8500 & $1,4 \%$ \\
Universidad Estatal de Milagro & 10000 & $1,7 \%$
\end{tabular}


Universidad Internacional del Ecuador

Universidad de Especialidades Espíritu Santo

Universidad Tecnológica Indoamérica

Universidad Andina Simón Bolívar

Universidad Particular Internacional SEK

Universidad Casa Grande

IDE Business School

Universidad del Pacífico Ecuador

ESPAE Graduate School Of Management ESPOL

Facultad Latinoamericana de Ciencias Sociales

Universidad de Los Hemisferios

Total

Fuente: Elaboración propia con base en métrica de Mentinno - Formación Gerencial. Con base en información disponible en LinkedIn Sales Navigator

En relación con las visitas a sitios web pueden encontrarse múltiples métricas con metodologías y variables diferentes, así como unidades de análisis. Para el efecto, SemRush y Alexa, utiliza variables independientes por cada una de las plataformas. En esta línea la Tabla XIII, evidencia dos sistemas de medición relevantes para los investigadores de mercados y para la publicidad digital en Internet.

\section{Tabla XIII. Sitios Web más visitados}

\begin{tabular}{c|ll} 
Posición & SemRush & Alexa \\
\hline 1 & wikipedia.org & google.com \\
2 & youtube.com & youtube.com \\
3 & facebook.com & elcomercio.com \\
4 & google.com & eluniverso.com \\
5 & google.com.ec & live.com \\
6 & whatsapp.com & google.com.ec \\
7 & live.com & facebook.com \\
8 & xnxx.com & zoom.us \\
9 & xvideos.com & ecuavisa.com \\
10 & pinterest.com & pichincha.com \\
\hline
\end{tabular}

Fuente: Elaboración propia con base en métrica de SemRush.com Ranking Ecuador

Resulta llamativo que de igual manera se comportan las plataformas tecnológicas de telefonía celular, no en la utilización de variables, sino en la diferencia de descargas de aplicaciones, lo que podría establecer una nueva categoría de segmentación o fragmentación de usuarios. Tal es el caso de Android y la plataforma de Apple iOS, como se advierte en la Tabla XIV. 
Tabla XIV. Aplicaciones descargadas en dispositivos móviles por plataforma en Ecuador

\begin{tabular}{c|l|l} 
Posición & \multicolumn{2}{|c}{ iOS Android } \\
\hline 1 & Disney + & FindNow \\
2 & WhatsApp Messenger & Kwai - Short Video \\
3 & ZOOM Cloud Meetings & Sticker.ly \\
4 & YouTube: Watch, Listen, Stream & Stretch Guy \\
5 & Facebook & YoungTunes \\
6 & TikTok & Among Us \\
7 & Messenger & Disney+ \\
8 & Instagram & Fat Pusher \\
9 & Gmail - Email by Google & Red Imposter \\
10 & Netflix & Foil Turning 3D \\
11 & Garena Free Fire-New Beginning & TikTok \\
12 & Spotify: Music and podcasts & StarMaker: Sing free \\
13 & Google Maps - Transit \& Food & VIX - CINE. TV \\
14 & Project Makeover & Parking Jam 3D \\
15 & Google & WhatsApp Messenger \\
16 & Microsoft Teams & Aplasta Palabras \\
17 & Snapchat & DOP 2 \\
18 & Pinterest & Garena Free Fire \\
19 & Telegram Messenger & Tiles Hop \\
20 & Google Chrome & ¡Cube Surfer! \\
\hline
\end{tabular}

Fuente: Elaboración propia con base en métrica de App Annie

En esta misma línea, se comportan las aplicaciones de compras en las dos plataformas. En la Tabla XV, se puede advertir que a pesar de que, cada una por su parte tiene un menú de aplicaciones exclusivas, logran un punto de encuentro relativo entre las aplicaciones originarias de otros proveedores. Así mismo, se observa como durante la Pandemia los sitios de alimentos y bebidas fueron significativamente utilizados por los usuarios de estas plataformas. 
Tabla XV. Aplicaciones de compras descargadas en dispositivos móviles por plataforma

\begin{tabular}{c|l|l} 
Posición & \multicolumn{1}{|c}{ Android } \\
\hline 1 & McDonald's App - Latinoamérica & Rappi \\
2 & Uber Eats & Uber Eats \\
3 & KFC App & KFC App \\
4 & Rappi & McDonald's App \\
5 & BEES Ecuador & Sweet\&Coffee \\
6 & Ice Cream Cake Maker & Domino's Pizza América Latina \\
7 & Coco Mercado & Domicilios.com \\
8 & Hornero Ecuador & Oahu Açai Bar \\
9 & Sweet\&Coffee & Vivino: Buy the Right Wine \\
10 & Domino's Pizza América Latina & Papa John's Pizza UAE \\
11 & Los Pollos de San Bartolo & Kobe Sushi \\
12 & Domino's Pizza & Hornero Ecuador \\
13 & Pizza Hut Delivery & Pizza Hut Delivery \\
14 & CookPad & DeUna Delivery \& Servicios \\
15 & Snap eats - Delivery & Zaymi \\
16 & Kobe Sushi & Intermittent Fasting App \\
17 & AQUA EC & Vaco y Vaca \\
18 & KFC Delivery Su & Snap eats - Delivery \\
19 & Unicorn Cake Maker & Pizza Hut CR \\
20 & Calorie, Carb \& Fat Counter & KFC Delivery Su \\
\hline
\end{tabular}

Fuente: Elaboración propia con base en métrica de Similar Web

Tabla XVI. Aplicaciones de alimentos y bebidas descargadas en dispositivos móviles por plataforma

\begin{tabular}{c|l|l} 
Posición & \multicolumn{2}{|c}{ Android } \\
\hline 1 & Alibaba.com & SHEIN \\
2 & OLX & Amazon Shopping \\
3 & Mercado Libre & Floryday \\
4 & Wish & OLX Classifieds \\
5 & Amazon Shopping & Mercado Libre \\
6 & SHEIN & Lyst \\
7 & Tipti & Wish \\
8 & Florydat & AliExpress \\
9 & PatPat & ZARA \\
10 & AliExpress & Apple Store \\
11 & eBay & eBay Shopping \\
12 & DHL LATAM eShop & Forever 21 \\
13 & Almacenes TIA & PULL\&BEAR \\
14 & Tiendamia & De Prati \\
15 & Amazon Assistant & Tipti \\
16 & Tiendeo & SuperEasy Ecuadpr \\
17 & SuperEasy Ecuadpr & PatPat \\
18 & Bershka & Tiendamia \\
19 & PULL\&BEAR & Bershka \\
20 & Fybeca & Cladwell \\
\hline
\end{tabular}

Fuente: Elaboración propia con base en métrica de Similar Web 


\section{DISCUSIÓN}

Resulta evidente que Ecuador se ha transformado en un ecosistema mediado por la interacción tecnológica. La disponibilidad de acceso a Internet ha permitido que sus habitantes puedan comunicarse a través de las diferentes plataformas de mensajería instantánea y de redes sociales. En los estudios del World Internet Project (2016) hasta el año 2015, solo el 2\% de los cibernautas había realizado algún tipo de compra por Internet. Esta constante se mantuvo hasta el año 2019, considerando que, a raíz de la pandemia, y de las dificultades para realizar compras físicas, este factor ascendió en 2020 al 10\% de los cibernautas. Este mismo año, se incrementó el 43,25\% de las ventas por este canal, con relación al año 2019, con un incremento en la frecuencia de compras del 21\%, según las métricas analizadas por Menntino en el presente informe. Esta dinámica se puede explicar desde la digitalización que realizaron los establecimientos físicos tradicionales, que vieron al e-commerce como una posibilidad de subsistencia y permanencia en el mercado, su respectiva presencia en el ambiente digital, de tal manera que se alcanzó en el último año 2.200 millones de dólares en ventas, con un crecimiento del $54 \%$ respecto al año 2019, y con preferencia de compras por WhatsApp del 49\%, Aplicaciones 44\% y Sitios Web 35\%.

A pesar de ser tipificado como un país en vía de desarrollo o del tercer mundo, Ecuador presenta indicadores significativamente altos de consumo de Internet y en especial de presencia en redes sociales, producto de la distribución demográfica de sus habitantes, de las políticas públicas de cobertura y acceso a Internet de los últimos 10 años por parte de los últimos dos gobiernos del país, lo que se enmarca en un ecosistema propio, con presencia de factores endógenos y exógenos, naturales y culturales, en la misma línea del enfoque la Ecología Humana de la Comunicación (López, Odriozola, Bernal, 2016).

Así mismo, responde a los enunciados de Sociedad de la Información, propuestos por Castells (2021), (1999), (2001), y de la Sociedad del Conocimiento de Bell (2000) en el que anuncia para la comunidad científica de la Comunicación, el advenimiento de nuevas arquitecturas tecnológicas que permitirán derribar las fronteras del espacio y del tiempo, García-Canclini (2001), y desplegarán las posibilidades comunicativas a través de la virtualidad, los flujos y las redes comunicacionales.

La explosión de las redes sociales podría no ser una novedad. En las generaciones anteriores de las diferentes sociedades, las redes se constituían por la vivienda, la cercanía geográfica, por la composición numerosa de las familias y en especial por la naturaleza comunicativa del ser humano, como ser social. Las relaciones sociales se suscitaban especialmente en la edad de la 
juventud, las cuales perdurarían a lo largo de la vida de las personas. Sin embargo, estas primeras redes sociales, eran limitadas por la localidad, a diferencia sustancial de las nuevas redes sociales, precisamente ampliadas por la globalidad, tal como lo señalara McLuhan, sobre la evolución hacia la conformación mediática de una Aldea Global (ed. 2020).

En esta línea, Islas y Gutiérrez (2003), interpretando a McLuhan, advierten sobre la reingeniería social que se viene desarrollando en la sociedad humana, a partir de las posibilidades telemáticas que presentan los nuevos medios de comunicación, en especial, por sus posibilidades de demarcación del espacio y del tiempo, en el que la tele educación, y el teletrabajo son posibles de desarrollar, Osicki (2012), como se ha demostrado durante el primer año de la Pandemia del COVID-19, y muy en contra posición de los grandes temores de las universidades, colegios, empresas, bancos, entre otros, en adoptar este tipo de metodologías de trabajos y educación virtual, López (2006).

\section{CONCLUSIONES}

En relación con los hábitos de consumo de nuevos medios digitales, se advierte que, en 2020, el primer año de la Pandemia del COVID-19, se incrementó significativamente la usabilidad de las redes sociales, los sitios web y las aplicaciones, no solo en los atributos comunicacionales, de información y de entretenimiento que se presentaron en los años anteriores, según los estudios del WIP, sino especialmente en las transacciones comerciales que se han venido desarrollando.

Este crecimiento ha sido determinante por la decisión que tomaron las empresas en la digitalización de presencia en estos nuevos medios digitales en Internet, y en efecto, por la confianza que las plataformas de pago ofrecieron a los consumidores, según los datos de las métricas analizadas, y que fue la principal causa de no compra en el pasado según los datos del WIP.

Ecuador, aún mantiene una brecha digital, a pesar del $80 \%$ de usuarios de Internet, lo que supone un $20 \%$ de personas, que podrían ser consideradas analfabetas digitales, no solo por la conectividad que supone, sino por la disponibilidad de la información, la comunicación a través de redes sociales, el entretenimiento y el comercio electrónico que podrían generar entre esta parte de la población.

Pese a que el $10 \%$ de la población de internautas ya realiza compras por Internet, y que su crecimiento fue del $500 \%$ en el último año, aún está pendiente un porcentaje mayor considerando que el $59 \%$ de ellos tienen más de 24 años, edad productiva de la población ecuatoriana. 
El 98\% de los internautas interactúan en redes sociales vía dispositivos móviles, de los cuales el 33\% están concentrados en Quito y Guayaquil, las dos ciudades más grandes del país. Esta dinámica supone una potencialidad comunicativa con las demás zonas del país, especialmente aisladas por su geografía, y un mercado potencial para la interacción comercial entre provincias. Las diferencias de la usabilidad de las aplicaciones en las dos plataformas telefónicas, iOS y Android, permite identificar una nueva forma de segmentación de audiencias y consumidores en el Ecuador, que podría mejorarse a partir de estudios cualitativos que caractericen al usuario de cada una de ellas.

Finalmente, la Pandemia del COVID-19 ha sido un acelerador significativo en la utilización de Internet, en el año 2020, por parte de las empresas que digitalizaron sus servicios y por parte de los consumidores que encontraron en las aplicaciones, las redes sociales, los sitios web y los sistemas de pago, la confianza necesaria para dinamizar el comercio electrónico ecuatoriano, como evidencia de la trasformación tecnológica de los nuevos medios de información digital que vivió en país en este año, más allá de la evolución natural de los procesos de apropiación cultural que viven las distintas sociedades de la humanidad.

\section{BIBLIOGRAFÍA}

Altheide, D. (1994). An Ecology of Communication: Toward a Mapping of the Effective Environment. The Sociological Quarterly 35(4), 665-683.

Banco Mundial (2020). Informe general. Washington DC: Banco Mundial.

Barbero, M. (2003). La globalización en clave cultural. Una mirada latinoamericana.

Renglones 53, 18-33.

Bell, D. (2000, January). Internet y la nueva tecnología. Retrieved from: http://www.letraslibres.com/revista/convivio/internet-y-la-nueva-tecnologia

Bringué, X., Sádaba, C. (2011). La generación interactiva en Ecuador. Quito: Ministerio de Telcomunicaciones y Sociedad de la Información.

Cabrera, M., Cupaiuoli, L. (2010). La influencia de Internet en la sociedad actual. Retrieved from:

http://www.solociencia.com/informatica/influencia-internet-sociedad-actual.htm

Cali, D.D. (2012). On Disciplining Media Ecology. Explorations in Media Ecology 10 (3-4), 335- 346.

Carpenter, E., McLuhan, M. (1956). The New Languages. Chicago Review 10(1), 46-52.

Castells, M. (2003). Internet, libertad y sociedad: una perspectiva analítica. Polis 1(4) Retrieved from http://www.redalyc.org/articulo.oa?id=30500410 
Castells, M. (2001). Internet y la Sociedad Red. Llicó inaugural del programa de doctorat sobre la societat de la informació i el coneixement. Barcelona: Universidad Oberta de $\begin{array}{lll}\text { Catalunya. } & \text { Retrieved }\end{array}$ http://www.uoc.edu/web/cat/articles/castells/castellsmain2.html

Castells, M. (1999). La Era de La información. Buenos Aires: Siglo XXI.

Dyjament, S. (2010). El uso de Internet en América Latina. Revista GPT: Gestión de Personas y Tecnología 7, 48-55.

García-Canclini, N. (2001). Culturas híbridas. Estrategias para entrar y salir de la modernidad. Buenos Aires: Paidós.

INEC (2019). Estadísticas de población. Quito: INEC.

Innis, H. (1999). The bias of communication. Toronto: University Toronto Press.

Internet World Stats (2011). Ecuador. Internet Usage and Market Report. Retrieved from http://www.internetworldstats.com/sa/ec.htm

Internet World Stats (2012). Ecuador. Internet Usage and Market Report. Retrieved from http://www.internetworldstats.com/sa/ec.htm

Internet World Stats (2015). Ecuador. Internet Usage and Market Report. Retrieved from http://www.internetworldstats.com/sa/ec.htm

Internet World Stats (2020). Ecuador. Internet Usage and Market Report. Retrieved from http://www.internetworldstats.com/sa/ec.htm

Islas, O., Gutiérrez, F. (2003). Ingeniería en comunicación social y en comunicación estratégica. Revista Latinoamericana de Comunicación CHASQUI. Retrieved from: http://chasqui.comunica.org/84/islas84.htm

Langer, S. (1967). An Introduction to Symbolic Logic. New York: Dover Publications.

López, J., Daniel, F.; Odriozola Chéné, J. \& Bernal Suárez, J.D. (2016). Theory of a Human Ecology of Communication. Empirical evidence of the Internet consumption ecosystem in Ecuador. Communication \& Society 29(1), 101-123.

López, J., Daniel, F. (2016). Ecología Humana de la Comunicación: análisis práctico para su comprensión. Comhumanitas, revista científica de Comunicación. 7 (1), 45-59.

López, J., Daniel, F.; Callejo, G.; Rodrigo, I.; Cajiao, E. (2013). Consumo de Internet en el Ecuador entre los años 2010 y 2012: hacia una ecología de la comunicación. ComHumanitas 4, 31- 45.

López, J., Daniel, F.; Eguiguren, M.J. (2011). Análisis comparativo del consumo de Internet en el Ecuador entre los años 2010-2011: más allá de la evolución, comportamientos 
significativos en la población de estudiantes, indicios de una "ecología de la comunicación". ComHumanitas 3, 123-154.

López, J., Daniel, F. (2010). Hábitos de consumo de Internet en Ecuador: diferencias significativas entre estudiantes. ComHumanitas 2, 61-93.

McLuhan, M., \& Ducher, P. (1996). Comprender los medios de comunicación: las extensiones del ser humano. Paidos, Barcelona.

McLuhan, M., \& Powers, B. R. (2020). La aldea global: transformaciones en la vida y los medios de comunicación mundiales en el siglo XXI. Editorial Gedisa

Mintel (2010). Reporte anual de estadísticas sobre las Tecnologías de Información. Quito: MINTEL.

Mintel (2011). Reporte anual de estadísticas sobre las Tecnologías de Información. Quito: MINTEL.

Mintel (2012). Reporte anual de estadísticas sobre las Tecnologías de Información. Quito: MINTEL.

Mintel (2016). Reporte anual de estadísticas sobre las Tecnologías de Información. Quito: MINTEL.

Mumford, L. (1967). The Myth of the Machine: Technics and human development. Michigan: Harcourt, Brace \& World.

Osicki, R.J. (2012). Is the Internet the New Temple? McLuhan Looks at Religion Looks at McLuhan. Explorations in Media Ecology 10(3-4), 347-357.

Postman, N. (June, 2000). The humanism of Media Ecology. In Proceedings of the Media Ecology Association. Inaugural Media Ecology Association Convention, New York, USA, (pp. 10-16).

Postman, N. (1988). Conscientious objections: stirring up trouble about language, technology, and education. New York: Knopf.

Scolari, C. (2008). Hipermediaciones: elementos para una teoría de la comunicación digital interactiva. Editorial Gedisa.

Schofield C.L. (2009). Theories: Mediatization and media ecology. In K. Lundby,

Mediatization: Concept, Changes, Consequences (pp. 83-98). New York: Peter Lang.

Infografía (Consultas realizadas entre el 4 y el 10 de enero de 2021)

Informe estadísticas del gobierno http://www.arcotel.gob.ec/estadisticas-2/

Informe estadísticas http://www.ecuadorencifras.gob.ec/.../tics\%202017_270718.pdf

Informe estadísticas datos móviles Gsma Intelligence Q42019 
https://www.gsma.com/mobileeconomy/latam-es/

Informe Alexa Top Sites Ecuador https://www.alexa.com/topsites/countries/EC

Informe Google Trends Ecuador https://trends.google.es/trends/yis/2020/ES/

Informe https://gs.statcounter.com/social-media-stats/all/ecuador

Informe https://www.rivaliq.com/blog/social-media-industry-benchmark-report/

Informe https://www.appannie.com/en/apps/ios/app/supereasy-ecuador/

Informe https://es.semrush.com/analytics/ranks/rank/?db=us

Informe https://www.similarweb.com/

Informe https://www.kantarworldpanel.com/co/Noticias/780-mil-hogares-compraron-por-e$\underline{\text { commerce-en-Ecuador }}$

Metrics Facebook Ads

Metrics Google Ads

Metrics Twitter Ads

Metrics Snapchat Ads

Metrics Linkedin Ads

Metrics Pinterest Ads

Metrics Instagram Ads

Metrics Messenger Ads 